\title{
Implantatgetragener Zahnersatz aus VITA ENAMIC IS
}

\section{Zur IDS 2015 wurden erstmals die VITA Implant Solutions-Rohlinge (IS) mit integrierter Schnitt- stelle zu einer Titan- / Klebebasis (z. B. TiBase) für implantatgetragene Kronenrekonstruktionen vorgestellt. PD Dr. Andreas Bindl (Abt. für Computergestützte Restaurative Zahnmedizin, Zent- rum für Zahnmedizin Universität Zürich und Praxis am Zürichberg, $\mathrm{CH}$ ) berichtet von einer Fall- versorgung mit VITA Enamics IS im Seitenzahnbereich.}

\section{Ausgangssituation}

Der 75-jährige Patient kam für die prothetische Versorgung zweier Implantate (Biomet 3i, Palm Beach Gardens, USA) in regio 25 und 26 in die Praxis. Nach 3-monatiger geschlossener Einheilung lagen gesunde Weichgewebeverhältnisse vor (๑ Abb. 1) und die Implantate waren vollständig osseointegriert ( $\bullet$ Abb. 2).

Es war geplant, die Implantate innerhalb 1 Behandlungssitzung freizulegen und prothetisch zu versorgen. Dies hat neben den allgemein mit der Chairside-Behand- lung verbundenen Zeit- und Kosteneinsparungen den Vorteil, dass keine wiederholte Manipulation des Weichgewebes erfolgt. Realisierbar ist diese Vorgehensweise erst seit der Einführung der CEREC Software-Version 4.2 (Sirona Dental, Wals, AT).

\section{Digitalisierung und Konstruktion} Zunächst wurden die Implantate freigelegt und kompatible Scanposts und Scanbodies (Sirona Dental) auf diesen fixiert (OAbb. 3 und 4). Der korrekte Sitz wurde mithilfe eines Röntgenbilds überprüft ( Abb.5). Danach erfolgte die digitale Abformung (CEREC Omnicam, Sirona Dental) der Situation mit Scanbodies, gefolgt von Scans des Antagonisten, sowie die bukkale Bissregistrierung. Das 3DModell wurde berechnet und die einteiligen Abutmentkronen unter Berücksichtigung aller relevanten Informationen mit der CEREC-Software V 4.4 konstruiert (O Abb. 6-8). 
Fertigung und Eingliederung

Nach dem Formschleifen in der CEREC MC

XL-Schleifeinheit wurden die IS-Kronen ausgearbeitet und mit den Instrumenten des VITA-Enamic-Polishing-Set technical extraoral poliert. Die Befestigung auf der TiBase erfolgte gemäß den Herstellerangaben mit Multilink Hybrid Abutment (Ivoclar Vivadent, Schaan, FL). Die Kronen zeigten gleich bei der Eingliederung eine präzise Passung ( $\odot$ Abb. 9 ) und fügten sich optisch gut in das Gesamtbild ein ( $\triangle \mathrm{Abb}$. 10 und 11). Die $\odot$ Abbildungen 12 und 13 zeigen das Resultat nach 6 Monaten. Mittlerweile hat sich eine straffe Gingivamanschette um beide Abutmentkronen ausgebildet.

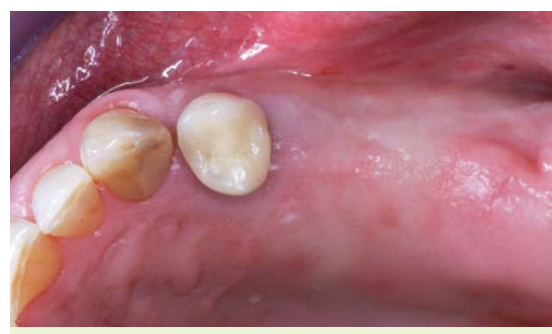

Abb. 1 Klinische Ausgangssituation nach 3-monatiger Einheilung der Implantate. Die Gingiva ist bereits anästhesiert, die Freilegung der Implantate kann erfolgen.

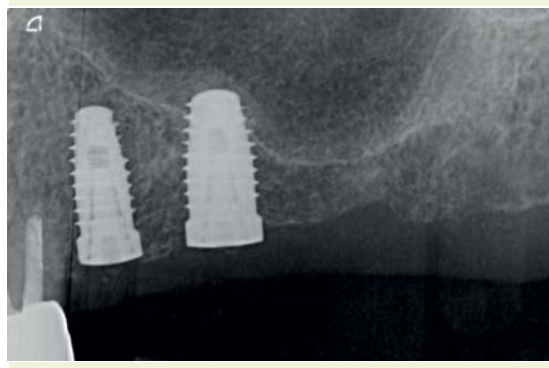

Abb. 2 Röntgenbild der beiden Implantate nach 3-monatiger Einheilung.

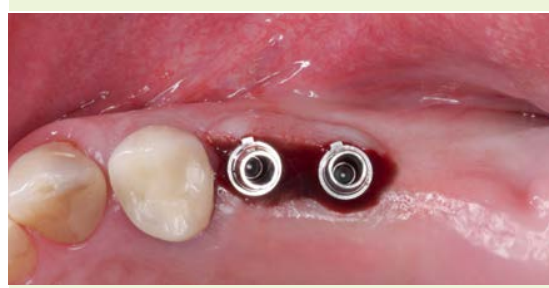

Abb. 3 Verschraubte Scanposts auf den freigelegten Implantaten.

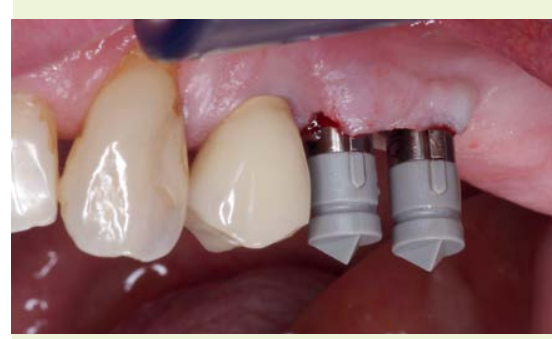

Abb. 4 Verschraubte Scanposts mit Scanbodies vorbereitet für die digitale Implantatabformung.

\section{Fazit}

Mit den IS-Rohlingen lässt sich innerhalb 1 Behandlungssitzung direkt verschraubter implantatgetragener Zahnersatz herstellen und eingliedern. Hybridkeramik weist zudem ein Elastizitäts-Modul auf, der dem von Dentin ähnlich ist. Inwieweit es dadurch zu einer Reduktion der auf das Implantat einwirkenden Kaukräfte kommt und ob sich dies positiv auf die Überlebensraten der implantatgetragener Rekonstruktionen auswirkt, wird in Studien untersucht.

\section{Korrespondenzadresse}

PD Dr. med. dent. A. Bindl

Station für Zahnfarbene- und ComputerRestaurationen

Universität Zürich

Attenhoferstr. 8a

$\mathrm{CH}-8032$ Zürich

andreas.bindl@zzm.uzh.ch

Dieser Beitrag entstand in Zusammenarbeit mit Vita Zahnfabrik GmbH, Bad Säckingen

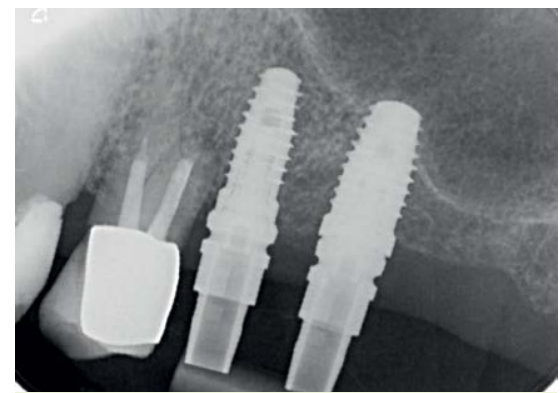

Abb. 5 Spaltfreier Sitz der Scanposts auf den Implantaten.

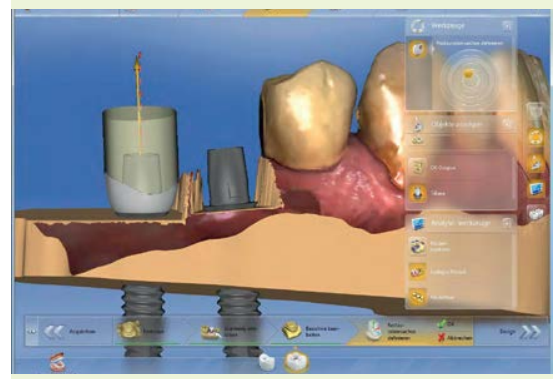

Abb. 6 Festlegung der Restaurationsachse für die computergestützte Konstruktion der Abutmentkronen.

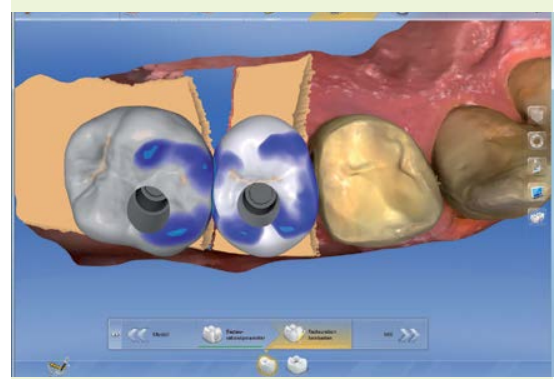

Abb. 7 Bearbeiten des vollanatomischen Konstruktionsvorschlags ...

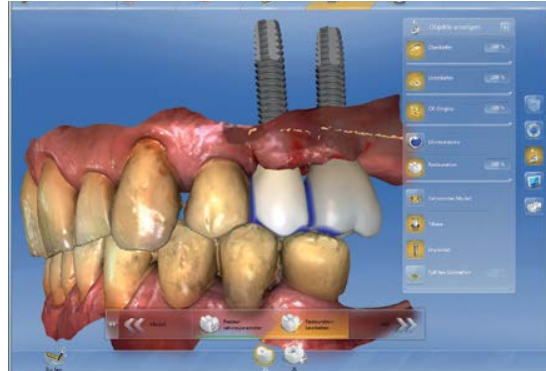

Abb. 8 ... unter Berücksichtigung der Mindeststärken und Okklusalkontakte.

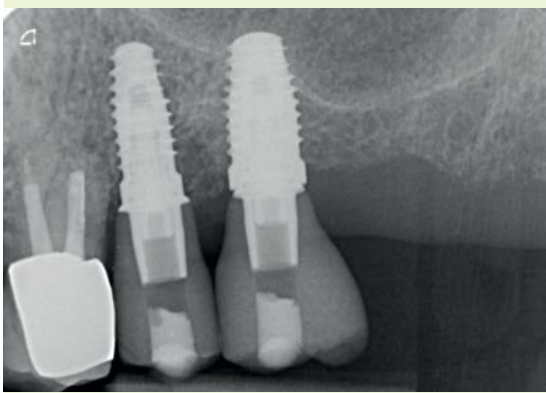

Abb. 9 Kontroll-Röntgenaufnahme nach Eingliederung der Versorgungen.

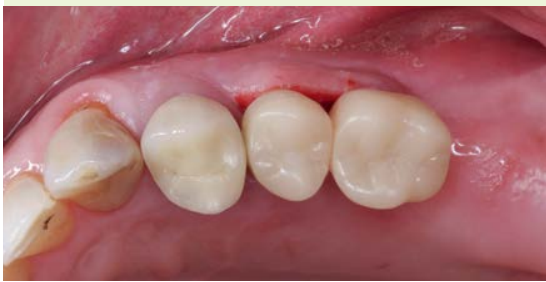

Abb. 10 Klinische Situation ...

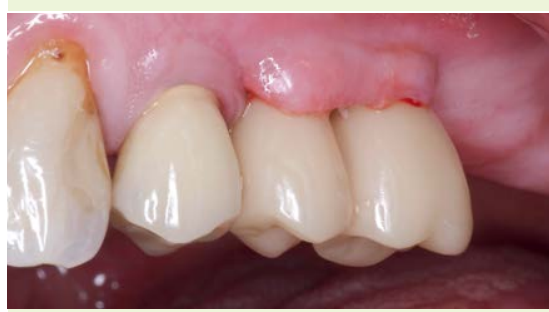

Abb. 11 ... unmittelbar nach Behandlungsabschluss.

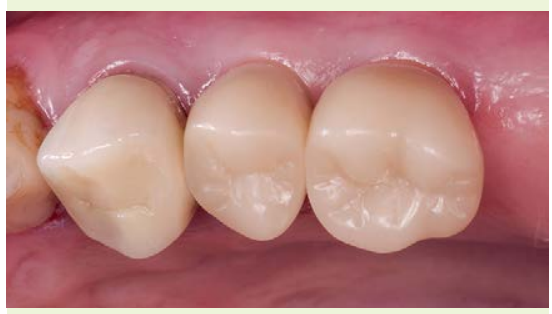

Abb. 12 Ergebnis ....

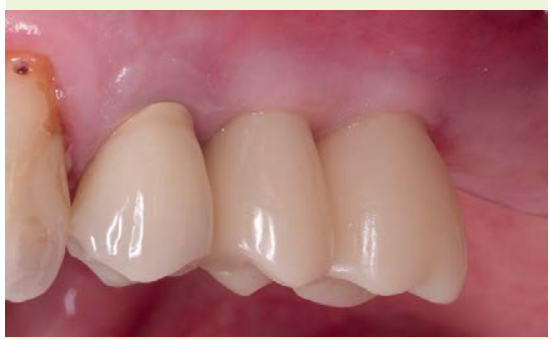

Abb. 13 ... 6 Monate nach der Eingliederung. 